\title{
Abre a mente PET: Formação crítica e cidadã promovidas pelos alunos do grupo PET Odontologia da Universidade Estadual de Maringá
}

Open your mind PET: Critical and citizen training promoted by students of the PET Dentistry group of Maringá State University

Abre la mente PET: Capacitación crítica y ciudadana promocionada por estudiantes del grupo PET Odontología de la Universidad Estatal de Maringá

Ana Elisa de Carvalho Manholer ORCID: https://orcid.org/0000-0002-7691-033X Universidade Estadual de Maringá, Brasil E-mail: anaelisamanholer@gmail.com Ana Flávia Borges Shimada ORCID: https://orcid.org/0000-0003-1637-8772 Universidade Estadual de Maringá, Brasil E-mail: anafshimada@gmail.com

Camila Fernanda Vasconcelos ORCID: https://orcid.org/0000-0002-1597-9448 Universidade Estadual de Maringá, Brasil E-mail: camilafv12@gmail.com

Carla Militão Ricken

ORCID: https://orcid.org/0000-0002-9174-590X Universidade Estadual de Maringá, Brasil E-mail: carlaricken7@gmail.com

Carlos Alberto Herrero de Morais ORCID: https://orcid.org/0000-0002-5856-5608 Universidade Estadual de Maringá, Brasil E-mail: carlosherrero31@gmail.com

Daniela Suemi Kamikawa

ORCID: https://orcid.org/0000-0002-7152-0500 Universidade Estadual de Maringá, Brasil

E-mail: danielasuemi97@gmail.com

Eduarda Letícia Pagliosa

ORCID: https://orcid.org/0000-0002-3160-7062 Universidade Estadual de Maringá, Brasil

E-mail: pagliosaeduarda@gmail.com

Flávia Akemi Nakayama Henschel ORCID: https://orcid.org/0000-0003-3186-3346 Universidade Estadual de Maringá, Brasil E-mail: flaviaakemi95@gmail.com

Gabriela Steckel Neme

ORCID: https://orcid.org/0000-0003-1986-0206 Universidade Estadual de Maringá, Brasil E-mail: gabrielasteckelneme@gmail.com

Laura Moretto Molina

ORCID: https://orcid.org/0000-0001-9815-7775 Universidade Estadual de Maringá, Brasil

E-mail: laurammolina1@gmail.com

Leonardo Galvão da Silva Garcia ORCID: https://orcid.org/0000-0002-5717-8040

Universidade Estadual de Maringá, Brasil E-mail: ra109875@uem.br

Letícia Caselato Ceron

ORCID: https://orcid.org/0000-0003-0818-2323

Universidade Estadual de Maringá, Brasil

E-mail: leticiacceron@gmail.com

Luisa Gonçalves Cardoso

ORCID: https://orcid.org/0000-0002-2182-707X

Universidade Estadual de Maringá, Brasil E-mail: luisagcardoso1@gmail.com 


\title{
Resumo
}

O Programa de Educação Tutorial (PET) tem o dever de propiciar aos alunos condições para a realização de atividades extracurriculares. Por isso, o objetivo deste artigo é apresentar o projeto de ensino intitulado: “Abre a Mente PET", desenvolvido pelos alunos do grupo PET Odontologia da Universidade Estadual de Maringá (UEM). Para a execução dessa atividade, os integrantes são divididos em grupos de quatro a cinco pessoas que apresentarão, em quatro dias distintos, temáticas variadas, que vão além dos ensinamentos contemplados durante a graduação. Cada equipe fica responsável por convidar uma pessoa que detém conhecimento teórico do assunto que irão apresentar, para que se tenha um melhor embasamento argumentativo e explicativo durante o debate. Em cada dia, o grupo responsável deve fazer a apresentação rápida, de forma expositiva e, posteriormente, é aberto um debate sobre o tema entre petianos (participantes do grupo PET) e membros da comunidade em geral, com a ajuda do convidado do grupo. As discussões promovidas até o presente momento foram enriquecedoras para o nosso grupo e comunidade, estimulando o senso crítico e trazendo conhecimentos que não estão contidos na grade curricular. Dessa forma, o projeto "Abre a Mente PET" representa um importante instrumento para desencadear mudanças na formação crítica e interdisciplinar dos envolvidos por meio de buscas por temas atuais que possuem relevância.

Palavras-chave: Capacitação profissional; Teoria crítica; Integração social.

\begin{abstract}
The Tutorial Education Program (PET) has the duty to provide students with conditions to carry out extracurricular activities. Therefore, the aim of this paper is to present the teaching project entitled: "Abre a Mente PET" (Open Your Mind PET), developed by the students of the PET Dentistry group of the State University of Maringá (UEM). For the execution of this activity, the members of the group are divided into groups of four to five people who will present, in four different days, varied themes, which go beyond the teachings contemplated during graduation. Each team is responsible for inviting a person who has theoretical knowledge of the subject they will present, so that they have a better argumentative and explanatory basis during the debate. Each day, the responsible group must make a quick presentation, in an expository way, and, afterwards, a debate on the theme is opened between PET members and members of the community in general, with the help of the group's guest. The discussions held so far have been enriching for our group and community, stimulating critical thinking and bringing knowledge that is not contained in the curriculum. In this way, "Abre a Mente PET" represents an important tool to trigger changes in the critical and interdisciplinary training of those involved, through searches for current topics that are relevant.
\end{abstract}

Keywords: Professional training; Critical theory; Social integration.

\section{Resumen}

El Programa de Educación Tutorial (PET) tiene la función de proporcionar a los estudiantes las condiciones para la realización de actividades extraescolares. Por ello, el objetivo de este artículo es presentar el proyecto docente titulado: "Abre a Mente PET" (Abre la Mente PET) desarrollado por los estudiantes del grupo de Odontología PET de la Universidad Estadual de Maringá (UEM). Para la realización de esta actividad, los integrantes del grupo se dividen en grupos de cuatro a cinco personas que presentarán, en cuatro días distintos, temáticas variadas, que van más allá de las enseñanzas contempladas durante la graduación. Y cada equipo se encarga de invitar a una persona que tenga conocimientos teóricos del tema que van a presentar, para que tenga una mejor base argumentativa y explicativa durante el debate. Cada día, el grupo responsable debe hacer una presentación rápida, de manera expositiva, y luego se abre un debate sobre el tema entre miembros del grupo PET y miembros de la comunidad en general, con la ayuda de un invitado del grupo. Las discusiones promovidas hasta el presente momento fueron enriquecedoras para el grupo 
y para la comunidad, estimulando el pensamiento crítico y trayendo conocimientos que no estan presentes en el plan de estudios habitual. Así, Abre a Mente PET representa una importante herramienta para desencadenar cambios en la formación crítica e interdisciplinar de los involucrados, a través de la búsqueda de temas actuales y relevantes.

Palabras clave: Capacitación profesional; Teoría crítica; Integración social.

\section{Introduçãa}

Um dos principais objetivos presentes nos Parâmetros Curriculares Nacionais é o desenvolvimento do senso crítico de maneira responsável e construtiva nas diferentes situações sociais, visando auxiliar o aluno a enfrentar o mundo atual como cidadão participativo, reflexivo e autônomo, conhecedor de seus direitos e deveres. Para isso, o ambiente acadêmico deve ser um espaço de formação e informação, favorecendo a inserção do aluno no dia a dia das questões sociais marcantes e em um universo cultural maior (Brasil, 1997). Além disso, o processo pedagógico, que visa a construção do saber do indivíduo, deve estimular o ato reflexivo, desenvolvendo sua capacidade de observação, análise crítica, autonomia de pensar e de ideias, ampliando os seus horizontes, tornando-o agente ativo nas transformações da sociedade, almejando interagir com a realidade na qual está inserido (Sordi \& Bagnato, 1998). O Programa de Educação Tutorial (PET) tem o dever de propiciar discussões de temas éticos, sócio-políticos, científicos e culturais relevantes para o País e/ou para o exercício profissional e para a construção da cidadania aos alunos da graduação (Brasil, 2006).

A discussão sobre a formação profissional passa a ser fundamental e estratégica quando falamos a respeito do SUS, o qual, sob a responsabilidade das três esferas de governo, a federal, a estadual e a municipal, operam o sistema de saúde, realizando ações e serviços em prol da saúde da população. Para tanto, os profissionais da saúde devem estar capacitados, do ponto de vista profissional, técnico, psicológico, político e humanístico. Sem essa qualidade de formação, o trabalhador da saúde não compreenderá essa área como uma produção social nem a complexidade do seu fazer a partir da escuta das necessidades da população (Silva \& Tavares, 2004).

Formar profissionais críticos compreende as metas propostas pelas instituições formadoras e dos que estão envolvidos com esta formação. No entanto, nem sempre é explicitado neste processo como desenvolver e atingir estes propósitos (Sordi \& Bagnato, 1998). Visto isso, e conforme publicado na Portaria no 976-2010-MEC e ratificado na Portaria $n^{\circ} 343-2013-M E C$, o Programa de Educação Tutorial (PET) tem o dever de propiciar aos alunos condições para a realização de atividades extracurriculares que complementem a sua formação acadêmica, procurando atender plenamente às necessidades do próprio curso de graduação, ampliar e aprofundar os objetivos e os conteúdos programáticos que integram sua grade curricular. Dessa forma, estimula-se o espírito crítico, bem como a atuação profissional pautada pela cidadania (Brasil, 2010).

Dessa forma, o grupo PET Odontologia da Universidade Estadual de Maringá (UEM) desenvolveu um projeto intitulado "Abre a Mente PET" o qual propõe elaborar momentos de discussão com a comunidade acadêmica sobre aspectos sociais, culturais, políticos e humanísticos, com o intuito de contribuir para a formação de profissionais de alta qualidade. Considerando essas informações, o objetivo deste artigo é apresentar o projeto de extensão "Abre a Mente PET" e debater como a inserção de discussões que estimulam a visão crítica tem contribuído para a formação dos acadêmicos.

\section{Metodologia}

O Abre a Mente PET é realizado em quatro encontros anuais, nos quais os integrantes do grupo PET Odontologia são divididos em quatro grupos de quatro a cinco pessoas, sendo mesclados nesses grupos alunos da $3^{\mathrm{a}}, 4^{\mathrm{a}}$ e $5^{\mathrm{a}}$ série. Cada equipe fica responsável por escolher um tema, apresentar e trazer um convidado que tenha um conhecimento teórico do assunto a ser abordado. Em cada apresentação, o grupo tem cerca de 20 minutos para fazer uma breve introdução do assunto de maneira expositiva. Posteriormente, é aberta uma roda de conversa com os petianos (participantes do grupo PET) e os demais participantes da comunidade em geral para debater sobre possíveis dúvidas e curiosidades sobre o assunto, com a ajuda do 
convidado do grupo, com um tempo de aproximadamente 40 minutos para essa discussão.

A atividade é previamente divulgada pelas mídias sociais do Grupo PET - Odontologia UEM, e qualquer pessoa, seja de outro curso, universidade ou comunidade externa é convidada a participar.

As rodas de conversa, segundo Moura e Lima (2014), consistem em um método de participação coletiva de debate acerca de determinada temática em que é possível dialogar com os sujeitos que se expressam e escutam seus pares e a si mesmos por meio do exercício reflexivo. Segundo Pereira et al. (2018), através da estratégia qualitativa da observação e troca de experiência, um dos seus objetivos é de socializar saberes e implementar a troca de de conversas, de divulgação e de conhecimentos entre os envolvidos, na perspectiva de construir e reconstruir novos conhecimentos sobre a temática proposta, como acontece no "Abre a Mente PET".

A universidade é a base do desenvolvimento científico e tecnológico, serve como pilar para o dinamismo científico nas sociedades atuais e é construída não apenas pelo ensino e pela pesquisa, mas principalmente pela extensão, como afirma Bartnik e Silva (2009). No entanto, no Brasil, Silva e Vasconcelos (2006) consideram que a educação superior prioriza o ensino e a pesquisa sem considerar as atividades de extensão como indispensáveis para a formação profissional, por isso a importância desse projeto está em alavancar esse pilar da instituição.

\section{Resultados e Discussão}

No ano de 2017, os temas abordados foram "Acessibilidade", "Intolerância Religiosa", "Feminismo" e "Cotas Raciais" com a presença de convidados que tinham conhecimento sobre o assunto. Já em 2018, os temas envolveram “Apropriação cultural", "Eutanásia", "Aborto", "Desarmamento", contando com a participação de dois médicos, uma professora de antropologia e um policial, respectivamente. Em 2019, foram apresentados quatro encontros da atividade Abre a mente PET, com os temas "Imigrantes e refugiados", "Investimentos", "Vegetarianismos e Veganismo" e "Influência da tecnologia sobre as crianças" e como convidados, um professor de história, um investidor, uma nutricionista, uma vegana e uma professora de psicologia se reuniram para participarem das discussões dos respectivos temas. O número total de participantes em 2019 foi de 82 pessoas, a maioria estudantes de odontologia, mas também houve participação de outros cursos.

Já em 2020, o Abre a mente PET tomou um formato diferente em vista da pandemia do Covid-19, foi realizado totalmente online, via plataforma Google Meet. Os temas foram "Odontologia: estereótipos e representatividade preta", "Consumo consciente", "Competitividade acadêmica: até que ponto é saudável?" e "Educação sexual”, como convidados: um dentista e um graduando pretos, uma idealizadora de produtos cosméticos sustentáveis, uma psicóloga especialista em terapia cognitivo-comportamental e uma professora de redação, e por fim, outra psicóloga, respectivamente. Neste ano, o número de inscritos foi de 192 pessoas, apresentando um aumento significativo em relação ao ano anterior, com participação de alunos de outras Universidades e cursos, inclusive de outros estados.

Como forma de ter uma resposta do público participante, no ano de 2020 disponibilizamos um formulário de satisfação para ser respondido ao final de cada roda de conversa, avaliando a atividade. Obtivemos 32 respostas por meio dos formulários, sendo que $100 \%$ das pessoas que responderam julgaram a atividade e os temas escolhidos como excelentes e, em relação à participação dos convidados, $75 \%$ avaliaram o conhecimento transmitido como excelente e $25 \%$ o classificaram como bom. O crescente número de participantes foi devido a divulgação que o grupo PET Odontologia realizou nas redes sociais e, também, pelo fato do Abre a mente PET ter acontecido no formato online, via plataforma Google Meet, o que possibilitou a participação de pessoas de outros estados. Os petianos avaliaram a atividade realizada como extremamente positiva, uma vez que trouxe discussões importantes para o grupo e para a comunidade, estimulando o senso crítico e, ainda, abrindo a mente para mais informações que não estão contidas na grade curricular. Foi possível uma troca de experiência entre 
os participantes, enriquecendo ainda mais o diálogo sobre o assunto discutido.

A metodologia de debater sobre assuntos extracurriculares é um processo de aprendizagem total, isto é, a integração de conhecimento. As atividades extracurriculares são consideradas integrativas por natureza, porque vinculam muitas áreas de conhecimento e experiência. Assim, o aluno consegue lidar com muitos aspectos importantes de um problema (Lunenburg, 2010). Segundo Pereira (2010), um dos componentes da criatividade é a expertise, composto por conhecimento técnico e intelectual. O conhecimento técnico é específico da área de atuação profissional, já o conhecimento intelectual é o conhecimento que está relacionado às áreas que fogem ao conhecimento da sua área de atuação, como conhecimento em poesia, música, cinema etc (Pereira, 2010) para os alunos de odontologia, por exemplo. Assim, as atividades extracurriculares proporcionam o desenvolvimento da capacidade crítica e construtiva do aluno, para que esse não seja apenas um mero repetidor de conceitos, mas que tenha desenvolvido a capacidade analítica de ver e compreender a realidade e a si mesmo de forma muito maior e mais reflexiva.

A participação do convidado é de grande importância, pois é a partir dele que as discussões e a roda de conversa contam com maiores embasamentos argumentativos. De acordo com Warschauer (2002) a roda de conversa é um momento singular de partilha, uma vez que pressupõe um exercício de escuta e fala, e os momentos de escuta são mais numerosos do que os de fala. As colocações de cada participante são construídas a partir da interação com o outro, seja para complementar, discordar ou para concordar com a fala anterior e por isso, os participantes devem estar cientes das informações que estão processando, discernindo entre argumentos válidos e falácias e consequentemente isso desenvolverá novas conexões para solucionar problemas e se conectarão com novos aprendizados (Barreiro, 2021).

Para os jovens universitários, há uma grande distância entre o que é ensinado na graduação e o que o mundo do trabalho exige. Grande parte do conteúdo dos cursos é de cunho teórico, e não prático, dificultando a aplicação do conhecimento na vida profissional (Vereguine, 2008). O mercado de trabalho, hoje em dia, exige que os profissionais consigam participar de novas experiências para se diferenciar, ressaltando a importância de participar de atividades extracurriculares, principalmente quando essas atividades acontecem dentro das próprias faculdades, pois são experiências que não fazem parte da grade curricular do curso superior e são fundamentais para complementar a formação acadêmica (Malschitzky, 2004). De acordo com Minarelli (1995), os profissionais precisam estar aptos do ponto de vista técnico, gerencial, intelectual, humano e social para solucionar, com rapidez, problemas cada vez mais sofisticados e específicos e, além disso, o mercado valoriza, dá preferência a profissionais com múltiplas habilidades e com iniciativa.

\section{Conclusão}

À luz do que foi exposto, entende-se que é de responsabilidade do Grupo PET Odontologia, contribuir para a melhoria da qualidade do curso de graduação e de propiciar uma melhor qualificação, formação profissional e cidadã. Dessa forma, a partir dos resultados obtidos, avaliamos a atividade desenvolvida como extremamente positiva. A ação proporciona, aos participantes, conteúdos e aprendizado além daqueles oferecidos pela Universidade, bem como leva conhecimentos diversos à comunidade. A discussão de assuntos relevantes, atualmente, permite um crescimento pessoal e representa um importante instrumento para desencadear mudanças na formação crítica e interdisciplinar dos envolvidos.

Diante da importância da atividade complementar reflexiva e do desenvolvimento crítico do cidadão, sugere-se que mais estudos sejam realizados no âmbito da formação crítica e social do acadêmico no intuito de estimular a formação de cidadãos completos. 
Research, Society and Development, v. 10, n. 4, e17010414005, 2021

(CC BY 4.0) | ISSN 2525-3409 | DOI: http://dx.doi.org/10.33448/rsd-v10i4.14005

\section{Referências}

Barreiro, M. P. R., Velásquez, B. I. H., Colamarco, B. I. L., Bravo, K. L. M., Barreiro, J. R. \& Rivadeneira, L. (202 1). El pensamiento crítico y su evaluación en la educación universitaria. Research, Society and Development 10 (3). https://doi.org/10.33448/rsd-v10i3.13748.

Bartnik, F. M. P., \& Silva, I. M. (2009). Avaliação da ação extensionista em universidades católicas e comunitárias. Avaliação: Revista da Avaliação da Educação Superior (Campinas), 14(2), 453-469. https://doi.org/10.1590/S1414-40772009000200010.

Brasil. Manual de orientações básicas. portal.mec.gov.br/pet.

Brasil. Ministério da Educação. Gabinete do Ministro. (2010, 28 de julho). Portaria n. ${ }^{\circ}$ 976, de 27 de julho de 2010 . Diário Oficial da União, Brasília, DF. p. 103 e 104.

Brasil. Ministério da Educação. Gabinete do Ministro. (2013, 25 de abril). Portaria n. 343 , de 24 de Abril de 2013 . Diário Oficial da União, Brasília, DF. p. 24.

Brasil. Secretaria de Educação Fundamental. (1997). Parâmetros curriculares nacionais: introdução aos parâmetros curriculares nacionais. Brasília, DF. http://portal.mec.gov.br/seb/arquivos/pdf/livro01.pdf.

Lunenburg, F. C. (2010). Extracurricular Activities. Sam Houston State University.

Malschitzky, N. (2004). Empregabilidade: um modelo para a instituição de ensino superior orientar e encaminhar a carreira profissional dos acadêmicos [Tese de Doutorado, Programa de Pós-Graduação em Engenharia de Produção, Florianópolis].

Minarelli, J. A. (1995). Empregabilidade: o caminho das pedras. Gente.

Moura, A. F., \& Lima, M. G. (2014). A reinvenção da roda: roda de conversa: um instrumento metodológico possível. Rev ista Temas em Educação, 23(1), 98106. https://periodicos.ufpb.br/index.php/rteo/article/view/18338.

Pereira, A. K., Koshino, M. F., Ferreira, T. R., \& Rocha, R. A. da. (2011). A importância das atividades extracurriculares universitárias para o alcance dos objetivos profissionais dos alunos de administração da universidade federal de santa catarina. Revista Gestão Universitária na América Latina. 163-194. https://doi.org/10.5007/1983-4535.2011v4nespp163.

Pereira, A. S., Shitsuka, D. M., Parreira, F. J. \& Shitsuka, R. (2018). Metodologia da Pesquisa Científica. UFSM.

Silva, J. P. V., \& Tavares, C. M. M. (2004). Integralidade: dispositivo para a formação crítica de profissionais de saúde. Revista Trabalho, Educação e Saúde, 2(2), 271-286. https://doi.org/10.1590/S1981-77462004000200004.

Silva, M. S., \& Vasconcelos, S. D. (2006). Extensão Universitária e formação profissional: avaliação da experiência das Ciências Biológicas na Universidade Federal de Pernambuco. Estudos em Avaliação Educacional, 17(33), 119-36. https://doi.org/10.18222/eae173320062130.

Sordi, M. R. L., \& Bagnato, M. H. S. (1998). Subsídios para uma formação profissional crítico-reflexiva na área da saúde: o desafio da virada do século. Revista Latino-Americana de Enfermagem, 6(2), 83-88. https://doi.org/10.1590/S0104-11691998000200012.

Veriguine, N. R. (2008). Autoconhecimento e informação profissional: implicações para o processo de planejar a carreira de jovens universitários. [Dissertação de Mestrado. Universidade Federal de Santa Catarina. Centro de Filosofia e Ciências Humanas. Programa de Pós-graduação em psicologia. Florianópolis, Brasil]. https://repositorio.ufsc.br/xmlui/handle/123456789/90875

Warschauer, C. (2002). Rodas em rede: oportunidades formativas na escola e fora dela. Paz e Terra. 